\title{
Indian kino tree (Pterocarpus marsupium): propagation, micropropagation, and biotechnology
}

\author{
Jaime A. Teixeira da Silva ${ }^{1 a}$, Mafatlal M. Kher ${ }^{2 b}$, Deepak Soner ${ }^{3 c}$, M. Nataraj ${ }^{2 \mathrm{~d}}$ \\ ${ }^{1}$ P. O. Box 7, Miki-cho post office, Ikenobe 3011-2, Kagawa-ken, 761-0799, Japan \\ ${ }^{2}$ P.G. Department of Biosciences, Sardar Patel University, Sardar Patel Maidan, Vadtal Rd., P.O. Box 39, Vallabh Vidyanagar, Gujarat, \\ 388120, India \\ ${ }^{3}$ Aakash Institute, Tribeni Complex, G.S. Road, Guwahati, Aasam, 781007, India \\ a,b,c,dCorresponding authors, E-mail: ajaimetex@yahoo.com; 'bmafatlalmkher@gmail.com; cdeepaksoner@gmail.com; \\ dmnatarajspu@gmail.com
}

\begin{abstract}
The Indian kino tree (Pterocarpus marsupium Roxb., Fabaceae) is listed in the IUCN red data list as a direct result of the excessive exploitation of its wood. Biotechnology has provided feasible and effective solutions for the tissue culture and mass micropropagation of $P$. marsupium, thus serving as a means to conserve important germplasm. The synthesis of information in this review aims to stimulate further research on P. marsupium. Breeding and biotechnological programmes that mass produce and effectively manage $P$. marsupium germplasm in vitro are required, using synthetic seed technology, cryopreservation and in situ conservation to manage this important wood germplasm. Molecular markers have been used to a limited extent to confirm the genetic stability of in vitro-propagated material. Biotechnological advances for this leguminous tree of commercial importance would benefit from research involving photoautotrophic micropropagation for improved rooting, bioreactors for the production of somatic embryos and secondary metabolites, thin cell layers for enhanced micropropagation, and cryoconservation including of synthetic seeds.
\end{abstract}

Key words: conservation, endangered species, in vitro conservation, IUCN red data list, Leguminoseae, medicinal plant, micropropagation, Pterocarpus marsupium, somatic embryogenesis.

Abbreviations: BA, 6-benzyladenine; IAA, indole-3-acetic acid; IBA, indole-3-butyric acid; MS, Murashige and Skoog; NAA, 1-napthaleneacetic acid.

\section{Introduction}

Pterocarpus (Leguminosae) is represented by 66 species globally (The Plant List 2018) that have high economic, medicinal and traditional value. Indian kino tree (Pterocarpus marsupium Roxb.), also known as Malabar kino or bijasal, is highly valued as a timber tree and for its pharmaceutically important gum kino (Badkhane et al. 2010; Mishra et al. 2013). It is a native plant of India, Nepal and Sri Lanka (Badkhane et al. 2010). P. marsupium is distributed in central, western and southern regions of India (Devgun et al. 2009).

\section{Improtance}

The heartwood of $P$. marsupium is an important source of pterostilbene (trans-3,5-dimethoxy-4-hydroxystilbene) (Mathew et al. 1977; Adinarayana, Syamasundar 1982; Dama et al. 1982; Manickam et al. 1997; Grover et al. 2005; Gupta, Gupta 2009; 2010; Chakraborty et al. 2010; Gupta, Gupta 2011; Joshi et al. 2012; Mohankumar et al. 2012). Pterostilbene has various clinical applications (Estrela et al. 2013; McCormack, McFadden 2013). In addition to pterostilbene, other secondary metabolites such as epicatechin (Adinarayana, Syamasundar 1982; Sheehan et al. 1983; Chakravarthy, Gode 1985), pterocarpol (Mathew et al. 1984; Mathew, Rao 1984; Rao et al. 1984; Maurya et al. 1985), pterosupin (Dama et al. 1982; Jahromi, Ray 1993; Manickam et al. 1997), pterocarposide (Handa et al. 2000; Maurya et al. 2004; Achari et al. 2012; Mishra et al. 2013) and marsuposides have also been discovered (Grover et al. 2004; Maurya et al. 2004; Gupta, Gupta 2009; Joshi et al. 2012; Mishra et al. 2013). Conventional uses, phytochemical extraction techniques, chemical constituents, pharmacological activity and commercial significance of $P$. marsupium have already been reviewed (Devgun et al. 2009; Badkhane et al. 2010; Hari, Gaikwad 2011), but no systematic review exists on the in vitro biotechnology of this species. $P$. marsupium is listed as a vulnerable plant in the IUCN red data list (IUCN 2017). In this review, we highlight how biotechnology, particularly in vitro technologies, can be used to preserve and sustainably multiply important germplasm, thus serving as a valuable tool for conservation purposes. 


\section{Sexual and vegetative propagation}

One of the simplest ways to mass propagate P. marsupium is via seed, although this can lead to a genetically heterogeneous population with variable growth characteristsics, which might not be a desired outcome for forestry or secondary metabolite production, which would preferably require clonal, and thus genettically uniform, material. Mishra et al. (2014) showed that seedlings of $P$. marsupium that emerged from large seeds ( 16 to $17 \mathrm{~mm}$ ) showed better growth and higher biomass than seedlings derived from medium-sized (13 to $15 \mathrm{~mm})$ and small (10 to $12 \mathrm{~mm}$ ) seeds. Patel and Patel (2016) reported 96.7\% seed germination of $P$. marsupium seeds on Murashige and Skoog (1962) medium when seeds were inoculated horizontally, and $90 \%$ of seedlings germinated in vitro survived in natural conditions when grown in a substrate of soil and manure (1:1). To provide elite germplasm for desired traits, especially phytochemicals or the wavy nature of the grain, vegetative propagation is desirable, and in vitro propagation allows for the production of true-to-type plants via micropropagation such as axillary shoot multiplication or shoot tip culture at a large scale, or somatic emberyogenesis, making plantlets with uniform characteristics continuously available. In vitro propagation also allows, in tree biotechnology, for the improvement of desired characteristics such as pathogen resistance or improved wood quality via genetic engineering (Rai, Shekhawat 2014), and in vitro propagation is frequently used for the large-scale propagation and conservation of various plants (Kher et al. 2016; Nataraj et al. 2016; Bi et al. 2017; Sanatombi, Sanatombi 2017; Teixeira da Silva et al. 2017). The next section provides an assessment of the progress made on the in vitro propagation of $P$. marsupium.

\section{In vitro propagation and tissue culture}

In vitro propagation, which has various advantages over vegetative or seed propagation and established micropropagation protocols, can be useful for various biotechnological applications but requires controlled environmental culture conditions. Thus, at each stage of in vitro propagation, effective, reproducible and cost-effective methods need to be standardized from cuture initiation to rooting and acclimatization to ensure viable plant production.

\section{Explants}

The source of explants, i.e., the mother plant, is a key initial requirement to initiate an in vitro propagation protocol. Surface sterilization is used to remove microbes from the surface of explants and thus it is essential to standardize the type of disinfectant, its concentration and the duration of sterilization to avoid infection by endophytic microorganisms that may reappear (Leifert et al. 1994).
Information about the explants used for the in vitro propagation of $P$. marsupium, as well as surface sterilization protocols, are summarised in Table 1. In general, explants are derived from either seedlings or mature plants (Das, Chatterjee 1993; Chand, Singh 2004; Tiwari et al. 2004; Anis et al. 2005; Husain et al. 2007; 2008; 2010; Tippani et al. 2013a; 2013b).

\section{Basal medium composition and culture conditions}

The most prefered basal medium for in vitro studies on $P$. marsupium is Murashige and Skoog (1962; MS) medium (Table 2). Chand and Singh (2004) noted that $0.8 \%$ agar in a water-based medium was sufficient for germination of $P$. marsupium seed while half-strength MS medium without plant growth regulators was used for in vitro seed germination of P. marsupium (Husain et al. 2007, 2008, 2010).

\section{In vitro propagation from predetermined meristems}

Three primary predetermined meristems have been employed in $P$. marsupium tissue culture: shoot tips, cotyledonary nodes and nodes from mature trees. Cotyldenory nodes were sucessfully applied for the in vitro propagation of P. marsupium (Chand, Singh 2004; Anis et al. 2005; Husain et al. 2007, 2008), most frequently employing 6-benzyladenine (BA), either alone or in combination with other cytokinins or auxins (Table 2).

\section{In vitro propagation (callogenesis, regeneration and somatic embryogenesis)}

Regeneration from immature P. marsupium zygotic embryos was possible on MS medium supplemented with $13.32 \mu \mathrm{M}$ BA and $2.85 \mu \mathrm{M}$ indole-3-acetic acid (IAA) (Tippani et al. 2013a). In another study by the same group (Tippani et al. 2013b), when immature cotyledons from nine-day old in vitro raised seedlings of $P$. marsupium were cultured on MS medium containing 1-napthaleneacetic acid (NAA), callus formed. When callus was subcultured onto MS medium containing BA and NAA, shoots developed from callus. Only one report is available on somatic embryogenesis of $P$. marsupium induced from hypocotyl segments from 12-day old in vitro seedlings (Husain et al. 2010; Table 2). Somatic embryogenesis is a useful method for obtaining clonal material that can serve as useful propagules for synthetic seed production, bioreactors and cryopreservation, especially for forestry species (Teixeira da Silva, Malabadi 2012).

\section{Rooting and acclimatization}

Sucessful roooting of in vitro raised plants followed by effective acclimatization and successful transfer of in vitro propagated plants to field conditions is the final objective of any micropopagation protocol and care is needed to avoid hyperhydricity in in vitro-raised plants, which tend to display poor rooting efficiency (Ruffoni, Savona 
Table 1. Explant source, size and surface sterilization procedures for preparation of tissue culture studies of Pterocarpus marsupium (chronological listing). No new tissue culture studies have been published after 2015. d, day(s); DW, distilled water; DDW, double distilled water; EtOH, ethyl alcohol (ethanol); $\mathrm{HgCl}_{2}$, mercuric chloride; IZE, immature zygotic embryo; $\mathrm{NaOCl}$, sodium hypochlorite; NR, not reported in the study; RTW, running tap water; s, second(s); SDW, sterilized (by autoclaving) distilled water; SW, sterilized water; y, year(s); TW, tap water

\begin{tabular}{|c|c|c|c|}
\hline Explant source & $\begin{array}{l}\text { Explant type, size and density; culture } \\
\text { vessel }\end{array}$ & Surface sterilization and preparation & Reference \\
\hline $\begin{array}{l}\text { Seeds from the wild } \\
\rightarrow \text { seedlings. Age of } \\
\text { mother plant NR. }\end{array}$ & $\begin{array}{l}\text { Cotyledonary node segments (size NR) } \\
\text { from } 20 \text {-d old seedlings. Test tubes/flasks } \\
\text { (1 explant/tube). }\end{array}$ & $\begin{array}{l}\text { Pods in DW } 24 \text {. Seeds: DW } 3-4 \times \rightarrow 0.1 \% \\
\text { Tween-20 } 15 \text { min } \rightarrow 0.06 \% \text { Savlon } 15 \text { min } \\
\rightarrow \text { DW } 3-4 \times \rightarrow 0.1 \% \mathrm{HgCl}_{2} 20 \mathrm{~min} \rightarrow 70 \% \\
\text { EtOH } 1 \mathrm{~min} \rightarrow 4-5 \times \mathrm{SDW}\end{array}$ & $\begin{array}{l}\text { Chand, Singh } \\
2004\end{array}$ \\
\hline $\begin{array}{l}\text { Seeds } \rightarrow \text { in vitro } \\
\text { seedlings ( } 4-5 \mathrm{~cm} \\
\text { tall, } 35-40-\mathrm{d} \text { old) }\end{array}$ & Nodal segments $(1.5-2 \mathrm{~cm})$. & $\begin{array}{l}\text { Seeds } \rightarrow \text { seed coat removed mechanically } \rightarrow \\
\text { soaked in SW overnight } \rightarrow 10 \% \mathrm{NaOCl} 10 \\
\min \rightarrow 3 \times \mathrm{SDW}\end{array}$ & $\begin{array}{l}\text { Tiwari et al. } \\
2004\end{array}$ \\
\hline $\begin{array}{l}\text { Seeds } \rightarrow \text { in vitro } \\
\text { seedlings ( } 5 \mathrm{~cm} \text { tall, } \\
18 \text {-d old). }\end{array}$ & $\begin{array}{l}\text { Seedling-derived cotyledonary nodes, } \\
\text { cotyledons, nodal segments, shoot tips (size } \\
\text { NR for all explants). Borosil test tubes ( } 1 \\
\text { explant/tube). }\end{array}$ & $\begin{array}{l}\text { Peeled seeds: RTW } 30 \mathrm{~min} \rightarrow \mathrm{DW} 24 \mathrm{~h} \rightarrow 5 \% \\
\text { Teepol (detergent) } 5 \mathrm{~min} \rightarrow \text { thorough wash in } \\
\mathrm{NR} \rightarrow 0.1 \% \mathrm{HgCl}_{2} 5 \mathrm{~min} \rightarrow 4-5 \times \mathrm{SDW}\end{array}$ & Anis et al. 2005 \\
\hline $\begin{array}{l}\text { Seeds } \rightarrow \text { in vitro } \\
\text { seedlings (6-, } 12-, 18- \\
\text { and } 24-\mathrm{d} \text { old). }\end{array}$ & $\begin{array}{l}\text { Seedling-derived cotyledonary nodes } \\
\text { of } 18 \text {-d-old seedlings }(1-2 \mathrm{~cm})(2007 \text {; } \\
2008) . \text { Hypocotyl segments }(0.5 \mathrm{~cm}) \text { from } \\
\text { 12-d-old seedlings } 2-4 \mathrm{~cm} \text { tall }(2010) .100 \text { - } \\
\text { mL Borosil test tubes ( } 1 \text { explant/tube). }\end{array}$ & $\begin{array}{l}\text { Peeled seeds: RTW } 30 \mathrm{~min} \rightarrow 5 \% \text { Teepol } 10 \\
\text { min } \rightarrow 1 \% \text { Bavistin (fungicide) } 15 \mathrm{~min} \rightarrow \\
\text { DW } 24 \mathrm{~h} \rightarrow 70 \% \text { EtOH } 30 \mathrm{~s} \rightarrow 0.1 \% \mathrm{HgCl}_{2} \\
5-6 \mathrm{~min} \rightarrow 5-6 \times \mathrm{SDW}\end{array}$ & $\begin{array}{l}\text { Husain et al. } \\
2007 ; 2008 ; 2010\end{array}$ \\
\hline $\begin{array}{l}\text { Seeds } \rightarrow \text { in vitro } \\
\text { seedlings (6-, } 12-, 18- \\
\text { and } 24-\mathrm{d} \text { old). }\end{array}$ & Seedling-derived cotyledonary nodes. & $\begin{array}{l}\text { Green pods: RTW } 20 \mathrm{~min} \rightarrow 1 \% \text { Laboline } 10 \\
\min \rightarrow \text { Tween- } 20 \text { (conc. NR) } 4 \mathrm{~min} \rightarrow \mathrm{TW} \rightarrow \\
0.1 \% \mathrm{HgCl}_{2} 15 \mathrm{~min} \rightarrow 5-6 \times \mathrm{SDW}\end{array}$ & $\begin{array}{l}\text { Porika et al. } \\
2009\end{array}$ \\
\hline $\begin{array}{l}\text { IZEs from seeds in } \\
\text { green pods } 4-9 \mathrm{w} \\
\text { after pollination. }\end{array}$ & $10 \times 8.5 \mathrm{~cm}$ bottles used & $\begin{array}{l}\text { Pods: RTW } 15 \mathrm{~min} \rightarrow 2 \% \text { Teepol } 10 \mathrm{~min} \rightarrow \\
5 \% \text { Tween-20 } 4 \mathrm{~min} \rightarrow 0.1 \% \mathrm{HgCl}_{2} 6 \mathrm{~min} \rightarrow \\
4-5 \times \mathrm{SDW}\end{array}$ & $\begin{array}{l}\text { Tippani et al. } \\
\text { 2013a }\end{array}$ \\
\hline $\begin{array}{l}\text { IZEs from seeds in } \\
\text { green pods from } \\
30-y \text {-old tree. }\end{array}$ & $\begin{array}{l}9 \mathrm{~d} \text { after IZE culture, immature cotyledons } \\
\text { cut and used as explants. Culture vessels } \\
\text { and explant density NR. }\end{array}$ & $\begin{array}{l}\text { Pods: RTW } 15 \mathrm{~min} \rightarrow 5 \% \text { Teepol gentle } \\
\text { shaking } 8 \mathrm{~min} \rightarrow 3-4 \times \mathrm{SDW} \rightarrow 0.1 \% \mathrm{HgCl}_{2} \\
8 \mathrm{~min} \rightarrow \text { repeated washes SDW. IZEs excised } \\
\text { and plated. }\end{array}$ & $\begin{array}{l}\text { Tippani et al. } \\
\text { 2013b }\end{array}$ \\
\hline $\begin{array}{l}\text { Node from } 10-y \text { old } \\
\text { tree }\end{array}$ & 1 node /100 mL flask (based on photos). & $\begin{array}{l}\text { NR in } 2013 \text { paper. } 2015 \text { paper: Tween- } 80 \\
5 \mathrm{~min} \rightarrow \mathrm{DW} \text { time } \mathrm{NR} \rightarrow 0.1 \% \text { Bavistin }+ \\
0.05 \% \text { streptomycin } 7 \mathrm{~min} \rightarrow \mathrm{DW} \text { time } \mathrm{NR} \rightarrow \\
0.1 \% \mathrm{HgCl}_{2} 7 \mathrm{~min} \rightarrow 3-4 \times \mathrm{SDW}\end{array}$ & $\begin{array}{l}\text { Jaiswal et al. } \\
2013 ; 2015\end{array}$ \\
\hline
\end{tabular}

2013). Rooting and acclimatization protocols for in vitro raised shoots of Pterocarpus species are summarized in Table 2. Only a few studies quantified the survival of micropropagated P. marsupium plants (Chand, Singh 2004; Husain et al. 2007; 2008; 2010; Tippani et al. 2013a; 2013b). The most effective auxin reported for rooting was indole3-butyric acid (IBA). Husain et al. (2008; 2007) reported the importance of phloroglucinol - an auxin-like rootinginducing compound (Teixeira da Silva et al. 2013) - in combination with IBA, for the rooting of $P$. marsupium shoots. Tippani et al. (2013a) pulsed $P$. marsupium shoots first in a solution of IBA for $24 \mathrm{~h}$ to induce in vitro roots using the same protocol to root putatively transgenic shoots (Tippani et al. 2013b).

\section{Genetic transformation}

Only a single report is available on the transient genetic transformation of $P$. marsupium by Agrobacterium tumefaciens (Tippani et al. 2013b). Callus was transformed with a hygromycin phosphotransferase $(h p t)$ gene (selectable marker) and intron-interrupted uidA (GUS) gene as the reporter gene under the control of the CaMV$35 \mathrm{~S}$ promoter.

\section{Molecular marker for clonal fiedility and phylogenetic relationships}

Molecular markers are useful tools for detecting somaclonal 
Table 2. In vitro conditions for tissue culture studies of Pterocarpus marsupium (chronological listing). AA, ascorbic acid; ABA, abscisic acid; AdS, adenine sulphate; AS, acetosyringone; AmS, ammomium sulphate; B5 medium, or Gamborg medium (Gamborg et al. 1968); $\mathrm{BA}, \mathrm{N}_{6}$-benzyladenine (BA) is used throughout even though BAP (6-benzylamino purine) may have been used in the original, according to Teixeira da Silva (2012b); cef, cefotaxime; CA, citric acid; CIM, callus induction medium; CWFT, cool white fluorescent tubes; d, day(s); FYM, farmyard manure; hyg, hygromycin; IAA, indole-3-acetic acid; IBA, indole-3-butyric acid; ISSR, inter-simple sequence repeat; IZE, immature zygotic embryo; Kn, kinetin (6-furfuryl aminopurine); MS, Murashige and Skoog, (1962) medium; NAA, $\alpha$-naphthaleneacetic acid; NR, not reported in the study; PG, phloroglucinol; PGR, plant growth regulator; PP, photoperiod; RH, relative humidity; RIM, root induction medium; SE, somatic embryo; SEIM, somatic embryo induction medium; SEMM, somatic embryo multiplication medium; SEM, shoot elongation medium; SG, seed germination; SIM, shoot induction medium; SMM, shoot multiplication medium; w, week(s); WPM, woody plant medium (Lloyd and McCown, 1980). * The original light intensity reported in each study has been represented since the conversion of lux to $\mu \mathrm{mol} \mathrm{m}{ }^{-2} \mathrm{~s}^{-1}$ is different for different illumination (main ones represented): for fluorescent lamps, $1 \mu \mathrm{mol} \mathrm{m}^{-2} \mathrm{~s}^{-1}$ $=80$ lux; the sun, $1 \mu \mathrm{mol} \mathrm{m}{ }^{-2} \mathrm{~s}^{-1}=55.6$ lux; high voltage sodium lamp, $1 \mu \mathrm{mol} \mathrm{m}{ }^{-2} \mathrm{~s}^{-1}=71.4$ lux (Thimijan, Heins 1983 ). ${ }^{* *}$ Even though calli was used in the original, the term callus has been used here based on recommendation of Teixeira da Silva (2012c). $\boldsymbol{g}$ Claims of somatic embryogenesis without sufficient proof (cytological, histological, genetic), i.e., only photos of macromorphology

\begin{tabular}{|c|c|}
\hline $\begin{array}{l}\text { Culture medium, PGRs, additives, } \\
\text { subcultures }\end{array}$ & Culture conditions * \\
\hline $\begin{array}{l}0.8 \% \text { agar }(\mathrm{SG}) . \mathrm{MS}+4.44 \mu \mathrm{M} \mathrm{BA}+0.26 \mu \mathrm{M} \\
\text { NAA (SIM). } 1 \frac{1}{2} \mathrm{MS}+9.84 \mu \mathrm{M} \text { IBA (RIM). } \mathrm{pH} \\
5.8 .2 \% \text { sucrose. } 0.8 \% \text { agar. }\end{array}$ & $\begin{array}{l}\text { 16-h PP. CWFT. } 40 \\
\mu \mathrm{mol} \mathrm{m}{ }^{-2} \mathrm{~s}^{-1} .24 \pm 2 \\
{ }^{\circ} \mathrm{C} .55-65 \% \text { RH. }\end{array}$ \\
\hline $\begin{array}{l}\text { PGR-free MS (SG, SIM). MS + } 13.31 \mu \mathrm{M} \text { BA } \\
+2.69 \mu \mathrm{M} \text { NAA }(\mathrm{SEM}) \cdot \mathrm{pH} 5.8 .3 \% \text { sucrose. } \\
0.8 \% \text { agar. }\end{array}$ & $\begin{array}{l}\text { 8-h PP. Light source } \\
\text { NR. } 55 \mu \mathrm{mol} \mathrm{m}{ }^{-2} \mathrm{~s}^{-1} \text {. } \\
25 \pm 2{ }^{\circ} \mathrm{C} . \mathrm{RH} \text { NR. }\end{array}$ \\
\hline
\end{tabular}

\section{Experimental outcome, maximum productivity, acclimatization and variation} $85 \%$ of cotyledonary nodes formed shoots (9.5/explant) after $15 \mathrm{w}$. About $30 \%$ of shoots 2004 formed roots after $25 \mathrm{~d}$. Acclimatization in autoclaved sand + peat moss + compost (1:1:1) with $52 \%$ survival. Range of SG\% (61-100\%), maximum on MS, Tiwari et al. lowest on White's (1963) basal medium and 2004 intermediate performance on B5 (78-80\%). Although 3.25 shoots/node formed in PGR-free MS, MS + $0.05 \mu \mathrm{M}$ IBA induced most nodes/shoot (4.95) and longest shoots $(2.92 \mathrm{~cm})$. Rooting was not described but successful (>68\%) acclimatization was claimed.

$\begin{array}{ll}\text { 1/2MS (SG). MS + } 5 \mu \mathrm{M} \text { BA (SIM). MS }+5 \mu \mathrm{M} & 16-\mathrm{h} \text { PP. CWFT. } \\ \mathrm{BA}+0.25 \mu \mathrm{M} \text { IAA (SEM). Pulse in } 200 \mu \mathrm{M} & \sim 1200 \text { lux. } 25 \pm 2{ }^{\circ} \mathrm{C} . \\ \text { IBA }+ \text { phenolic acid } 5 \rightarrow 1 / 2 \mathrm{MS}+0.5 \mu \mathrm{M} \text { IBA } & 55-60 \% \text { RH. } \\ \text { (RIM). Subcultures NR.pH 5.8. } 3 \% \text { sucrose. } & \\ 0.8 \% \text { agar. } & \end{array}$

PGR-free $1 \frac{1}{2} \mathrm{MS}$ (SG). MS + $0.4 \mu \mathrm{M}$ TDZ

(SIM). Subculture every $21 \mathrm{~d}$. MS $+5 \mu \mathrm{M}$ BA (SEM). Pulse in $200 \mu \mathrm{M}$ IBA $4 \mathrm{~d}$ paper bridge $\rightarrow 1 / 2 \mathrm{MS}+0.2 \mu \mathrm{M}$ IBA $+0.96 \mu \mathrm{M}$ PG $+2 \%$ sucrose (RIM). pH 5.8. $3 \%$ sucrose. $0.7 \%$ agar.

16-h PP. CWFT. 50
$\mu \mathrm{mol} \mathrm{m}{ }^{-2} \mathrm{~s}^{-1} .25 \pm 2$
${ }^{\circ} \mathrm{C} .50-60 \%$ RH.
\% SG NR. 7.8 shoots/cotyledonary node. $40-50 \%$ of shoots rooted. Acclimatized plants shown, but details NR. $80 \%$ SG in $4-5$ d. 15.2 shoots/cotyledonary node in $90 \%$ of explants. $65 \%$ of shoots rooted (chlorogenic acid and salicylic acid were not as effective as PG). 70\% survival after acclimatization in autoclaved soil + Soilrite $^{\oplus}(1: 1)$ at same conditions as in vitro plants for 2 months.

\begin{tabular}{|c|c|}
\hline $\begin{array}{l}\text { PGR-free } 1 \frac{1}{2} \mathrm{MS} \text { (SG). MS }+4 \mu \mathrm{M} \text { BA }+0.5 \mu \mathrm{M} \\
\text { IAA }+20 \mu \mathrm{M} \text { AdS (SIM). Subculture every } \\
21 \mathrm{~d} . \mathrm{MS}+5 \mu \mathrm{M} \text { BA (SEM). Pulse in } 100 \mu \mathrm{M} \\
\text { IBA }+15.84 \mu \mathrm{M} \text { PG } 7 \mathrm{~d} \rightarrow 1 / 2 \mathrm{MS}+2 \% \text { sucrose } \\
\text { (RIM).pH 5.8. } 3 \% \text { sucrose. } 0.7 \% \text { agar. }\end{array}$ & $\begin{array}{l}\text { 16-h PP. CWFT. } 50 \\
\mu \mathrm{mol} \mathrm{m}{ }^{-2} \mathrm{~s}^{-1} .25 \pm 2 \\
{ }^{\circ} \mathrm{C} .55-65 \% \text { RH. }\end{array}$ \\
\hline $\begin{array}{l}\mathrm{MS}+4.44 \mu \mathrm{M} \text { BA }(\mathrm{SIM}) .1 / 2 \mathrm{MS}+49 \mu \mathrm{M} \text { IBA } \\
(\mathrm{RIM}) . \mathrm{pH} 5.8 .3 \% \text { sucrose. } 0.8 \% \text { agar. }\end{array}$ & $\begin{array}{l}\text { 16-h PP. CWFT. } \\
40-60 \mu \mathrm{mol} \mathrm{m}{ }^{-2} \mathrm{~s}^{-1} \text {. } \\
25 \pm 2{ }^{\circ} \mathrm{C} . \mathrm{RH} \text { NR. }\end{array}$ \\
\hline
\end{tabular}
\% SG NR. 8.6 shoots/cotyledonary node in $85 \%$ of explants. MS formed more shoots than on B5 or WPM. 70\% of shoots rooted. $75 \%$ survival after acclimatization in Soilrite ${ }^{\circ}$ and watered with $1 / 4 \mathrm{MS}$. SG not an objective of the study, so NR. 12.9 shoots/immature seed formed on SIM ( $3.8 \mathrm{~cm}$ long). $68 \%$ of shoots rooted in RIM (2.1 roots/shoot). $75 \%$ plantlets survival after acclimatization in sterilized soil + vermiculite (1:1) Anis et al.

\section{Reference}

Chand, Singh Tiwa

Anis et al.
2005
Husain et al. 2007 2008 Porika et al. 2009
Husain et al. 
Table 2. continued

\begin{tabular}{|c|c|c|c|}
\hline $\begin{array}{l}\text { Culture medium, PGRs, additives, } \\
\text { subcultures }\end{array}$ & Culture conditions * & $\begin{array}{l}\text { Experimental outcome, maximum } \\
\text { productivity, acclimatization and variation }\end{array}$ & Reference \\
\hline $\begin{array}{l}\text { PGR-free } 1 / 2 \mathrm{MS}(\mathrm{SG}) . \mathrm{MS}+5 \mu \mathrm{M} 2,4-\mathrm{D}+1 \\
\mu \mathrm{M} \text { BA (CIM). MS }+2 \mu \mathrm{M} \text { BA (SEIM). MS } \\
+0.5 \mu \mathrm{M} \text { BA }+0.1 \mu \mathrm{M} \text { NAA }+10 \mu \mathrm{M} \text { ABA } \\
\text { (SEMM). } 1 \frac{1}{2} \mathrm{MS}+1 \mu \mathrm{M} \text { BA (SE germination). } \\
\text { pH } 5.8 .3 \% \text { sucrose. } 0.7 \% \text { agar. }\end{array}$ & $\begin{array}{l}\text { 16-h PP. CWFT. } 50 \\
\mu \mathrm{mol} \mathrm{m}{ }^{-2} \mathrm{~s}^{-1} \cdot 25 \pm 2 \\
{ }^{\circ} \mathrm{C} .50-60 \% \text { RH. }\end{array}$ & $\begin{array}{l}90 \% \text { of hypocotyls formed callus. } 23 \text { globular } \\
\text { stage SEs/callus clump after } 4 \text { w. } 60 \% \\
\text { survival after acclimatization in Soilrite and } \\
\text { watered with } 1 / 4 \mathrm{MS} \text {. }\end{array}$ & $\begin{array}{l}\text { Husain et al. } \\
2010^{* *}\end{array}$ \\
\hline $\begin{array}{l}\text { MS + } 13.32 \mu \mathrm{M} \text { BA }+2.85 \mu \mathrm{M} \text { IAA (SIM). MS } \\
+4.44 \mu \mathrm{M} \text { BA (SEM). Subcultures every } 3 \mathrm{w} . \\
14.66 \mu \mathrm{M} \text { IBA } 24 \mathrm{~h} \rightarrow \frac{1}{2} \mathrm{MS} \text { (RIM). pH 5.8. } 3 \% \\
\text { sucrose. } 0.8 \% \text { agar. }\end{array}$ & $\begin{array}{l}\text { 16-h PP. CWFT. } \\
10-15 \mu \mathrm{mol} \mathrm{m}^{-2} \mathrm{~s}^{-1} \text {. } \\
25 \pm 2{ }^{\circ} \mathrm{C} .50-60 \% \\
\text { RH. }\end{array}$ & $\begin{array}{l}93.8 \% \text { of IZEs formed } 17.3 \text { shoots/IZE, or } \\
27.2 \text { shoots/IZE after subculture. BA induced } \\
\text { more shoots than Kin. } 70.8 \% \text { of shoots } \\
\text { rooted after } 4 \text { w. } 74 \% \text { of plantlets survived } \\
\text { after acclimatization in sterilized soil + } \\
\text { vermiculite ( } 1: 1) \text {, irrigated with } 1 / 10 \text { dilution } \\
\text { of liquid MS, hardened, then transferred to } \\
\text { garden soil + sand ( } 1: 1) \text {. ISSR was used to } \\
\text { confirm genetic stability of in vitro-derived } \\
\text { plantlets relative to mother plants and } \\
\text { acclimatized plants. }\end{array}$ & $\begin{array}{l}\text { Tippani et al. } \\
\text { 2013a }\end{array}$ \\
\hline 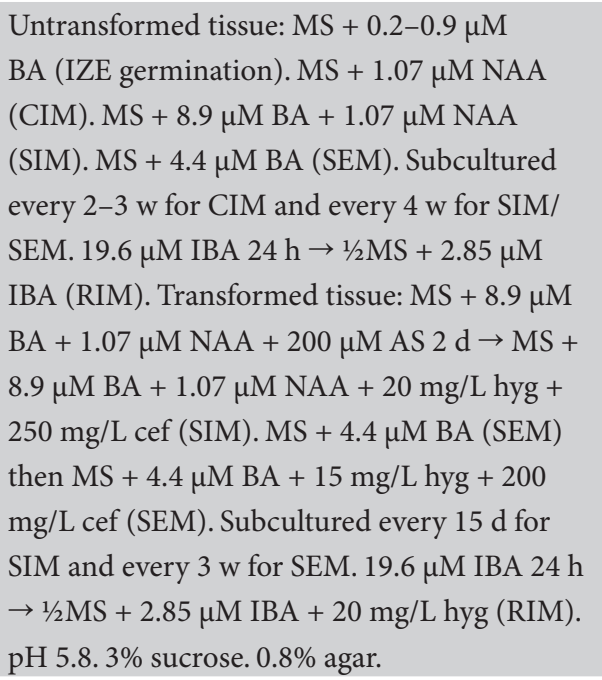 & 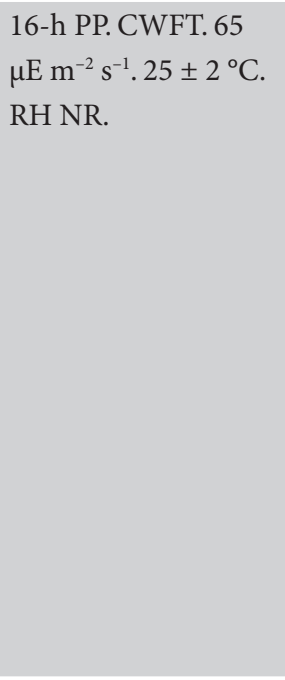 & $\begin{array}{l}\text { Callus formed in } 2 \mathrm{w} \text { from cotyledons } \\
\text { derived from seedlings (in vitro germinated } \\
\text { IZEs). } 60.4 \% \text { of cotyledons formed callus } \\
\text { and } 12.2 \text { shoots/callus cluster after } 4 \mathrm{w} \text {. } \\
75 \% \text { of shoots rooted after } 4 \mathrm{w} \text {, forming } 4.5 \\
\text { roots/shoot. } 95 \% \text { of plantlets survived after } \\
\text { acclimatization in vermiculite + perlite ( } 1: 1 \text { ), } \\
\text { irrigated with } 1 / 2 \mathrm{MS} .20 .9 \% \text { transformation } \\
\text { efficiency claimed (see text for more details } \\
\text { of the transformation experiments). }\end{array}$ & $\begin{array}{l}\text { Tippani et al. } \\
2013 b^{* *}\end{array}$ \\
\hline $\begin{array}{l}\mathrm{MS}+\mathrm{Kn} \text { (conc. NR) + additives }(568 \mu \mathrm{M} \text { AA } \\
+260 \mu \mathrm{M} \mathrm{CA}+605 \mathrm{AmS}+217 \mu \mathrm{M} \text { AdS }), \mathrm{pH} \\
\text { 5.8. } 0.8 \% \text { agar. Carbon source NR. }\end{array}$ & $\begin{array}{l}\text { 16-h PP. CWFT. } 1600 \\
\text { lux. } 26 \pm 2{ }^{\circ} \mathrm{C} .\end{array}$ & $\begin{array}{l}\text { About } 6 \text { shoots } 1.5 \mathrm{~cm} \text { long obtained from } \\
\text { single nodes. All shoots were healthy without } \\
\text { any necrosis. Acclimatization NR. }\end{array}$ & $\begin{array}{l}\text { Jaiswal et al. } \\
2013\end{array}$ \\
\hline $\begin{array}{l}\text { MS + } 13.95 \mu \mathrm{M} \text { Kn + additives (Jaiswal et al. } \\
\text { 2013) (SIM). MS + 9.3 } \mu \mathrm{M} \text { Kn + 0.54 } \mu \mathrm{M} \text { NAA } \\
\text { + additives (Jaiswal et al. 2013) (SMM). MS } \\
+4.92 \mu \mathrm{M} \text { IBA (RIM). pH 5.8. 0.8\% agar. 3\% } \\
\text { sucrose. }\end{array}$ & $\begin{array}{l}\text { 16-h PP. CWFT. } 1600 \\
\text { lux. } 26 \pm 2{ }^{\circ} \mathrm{C} \text {. }\end{array}$ & $\begin{array}{l}\text { In SIM, } 2.51 \text { shoots/node (1.1- } 1.9 \text { shoots/ } \\
\text { node when BA was used) in } 64.4 \% \text { of nodes. } \\
\text { In SMM, } 5.0 \text { shoots/node. } 42.2 \% \text { of shoots } \\
\text { rooted. Acclimatization in sand, soil and } \\
\text { FYM (1:1:1). }\end{array}$ & $\begin{array}{l}\text { Jaiswal et al. } \\
2015\end{array}$ \\
\hline
\end{tabular}

variation in in vitro culture and have many applications, including the definition of phylogenetic relationships, construction of genetic linkage maps, QTL identification, genome mapping, association mapping and linkage disequilibrium, marker-assisted breeding and genomewide marker-assisted selection, genetic diversity analysis for crop breeding, comparative genomics and taxonomic classification (Cloutier, Landry 1994; Schlötterer 2004; Teixeira da Silva et al. 2007a; Kalia et al. 2011; Hayward et al. 2015). Despite these strengths, most of these issues have not yet been explored in Pterocarpus. Tippani et al. (2013a) used ISSR markers to detect variation in plants raised from immature P. marsupium zygotic embryos. SaslisLagoudakis et al. (2011) used plastid regions $r b c L$ and matK, as barcodes for two Pterocarpus species, and $n d h F-$ rpL32 as a plastid marker, as an intergenic spacer to scan the plastid genome; amplified $n r I T S 2$ and $t r n L-F$ intergenic spacers were useful to elucidate phylogenetic relationships. 
Genetic differences in P. officinalis populations between island and continental populations were discovered using AFLP markers (Rivera-Ocasio et al. 2002; 2006). Muller et al. (2006) identified chloroplast and nuclear microsatellite markers for $P$. officinalis, which they then used to study genetic diversity and gene flow. Molecular markers have a solid base of use for select Pterocarpus species, and can thus serve as a platform for further applied molecular studies.

\section{Conclusions and future perspectives}

This review highlights the key advances in the tissue culture-based biotechnology of P. marsupium. To date, effective protocols for seed surface disinfection and in vitro germination exist. There are also effective protocols for direct shoot regeneration from a range of explants, or through callus induction. Rooting and acclimatization protocols are also well established. Only a single report on somatic embryogenesis - itself without sufficient proof that the structures obtained were in fact somatic embryos - indicates that this area of tissue culture still needs much improvement.

Even though only a single genetic transformation study exists for P. marsupium, a reliable and reproducible in vitro culture protocol will assist researchers in seeking transgenic strategies to fortify $P$. marsupium germplasm against abiotic and biotic stresses, induce more rapid growth, or resistance to pests and diseases. To fortify current tissue culture efforts, several other strategies can, and should, be attempted: photoautotrophic micropropagation (Xiao et al. 2010), bioreactors for large-scale production of somatic embryos and enhancement of secondary metabolites (Ziv 2005; Sharma, Sharma 2009; Huang, McDonald 2012), thin cell layers to enhance micropropagation and quantitative organogenesis (Nhut et al. 2003; Teixeira da Silva 2003; Teixeira da Silva et al. 2007b; Teixeira da Silva 2012a; Teixeira da Silva, Dobránszki 2013; 2014). The ability to stably produce units that would allow for germplasm conservation would then stimulate the need for cryoconservation (Berjak et al. 2011; Benelli et al. 2013; Kulus, Zalewska 2014; Teixeira da Silva et al. 2015; Bi et al. 2017), including through the application of synthetic seeds (Rai et al. 2009; Sharma et al. 2013).

\section{Acknowledgements}

The authors thank Dr. Emilia Caboni (Agricultural Research Council (CRA), Fruit Tree Research Centre, Rome, Italy), Dr. Randall Niedz (U.S. Department of Agriculture, Agricultural Research Service, U.S. Horticultural Research Laboratory, FL, USA) and Dr. Ivana Gribaudo (Istituto Protezione Sostenibile delle Piante - CNR, Grugliasco, Italy) for ideas, comments and suggested improvements to an early version of the manuscript.

\section{Authors' contribution and conflicts of interest statement}

All authors contributed equally to all aspects of review develop- ment and writing. The authors have no conflicts of interest to declare.

\section{References}

Achari B., Dutta P.K., Roy S.K., Chakraborty P., Sengupta J., Bandyopadhyay D., Maity J.K., Khan I. A., Ding Y., Ferreira D. 2012. Fluorescent pigment and phenol glucosides from the heartwood of Pterocarpus marsupium. J. Nat. Prod. 75: 655-660.

Adinarayana D., Syamasundar K.V. 1982. A new sesquiterpene alcohol from Pterocarpus marsupium. Phytochemistry 21: 1083-1085.

Anis M., Husain M.K., Shahzad A. 2005. In vitro plantlet regeneration of Pterocarpus marsupium Roxb., an endangered leguminous tree. Curr. Sci. 88: 861-863.

Badkhane Y., Yadav A.S., Sharma A.K., Raghuwanshi D.K., Uikey S.K., Mir F.A., Lone S.A., Murab T. 2010. Pterocarpus marsupium Roxb. biological activities and medicinal properties. Int. J. Adv. Pharm. Sci. 1: 350-357.

Benelli C., De Carlo A., Engelmann F. 2013. Recent advances in the cryopreservation of shoot-derived germplasm of economically important fruit trees of Actinidia, Diospyros, Malus, Olea, Prunus, Pyrus and Vitis. Biotechnol. Adv. 31: 175-85.

Berjak, P., Bartels, P., Benson, E.E., Harding, K., Mycock, D.J., Pammenter, N.W., Sershen, Wesley-Smith, J., 2011. Cryoconservation of South African plant genetic diversity. In Vitro Cell. Dev. Biol. Plant 47: 65-81.

Bi W.-L., Pan C., Hao X.-Y., Cui Z.-H., Kher M.M., Marković Z., Wang Q.-C., Teixeira da Silva J.A. 2017. Cryopreservation of grapevine (Vitis spp.) - a review. In Vitro Cell. Dev. Biol. Plant 53: 449-460.

Chakraborty A., Gupta N., Ghosh K., Roy P. 2010. In vitro evaluation of the cytotoxic, anti-proliferative and antioxidant properties of pterostilbene isolated from Pterocarpus marsupium. Toxicol. In Vitro 24: 1215-1228.

Chakravarthy B.K., Gode K.D. 1985. Isolation of (-)-epicatechin from Pterocarpus marsupium and its pharmacological actions. Planta Med. 51: 56-59.

Chand S., Singh A.K. 2004. In vitro shoot regeneration from cotyledonary node explants of a multipurpose leguminous tree, Pterocarpus marsupium Roxb. In Vitro Cell. Dev. Biol. Plant 40: 167-170.

Cloutier S., Landry B.S. 1994. Molecular markers applied to plant tissue culture. In Vitro Cell. Dev. Biol. Plant 30: 32-39.

Dama A., Syamasundar K.V., Seligmann O., Wagner H. 1982. Structure elucidation of pterosupin from Pterocarpus marsupium, the first naturally occurring C-glycosyl-Bhydroxy-dihydrochalcone. Verlag Zeit. Naturforsch. 37: 145147.

Das T., Chatterjee A., 1993. In vitro studies of Pterocarpus marsupium - an endangered tree. Indian J. Plant Physiol. 36: 269-272.

Devgun M., Nanda A., Ansari S.H. 2009. Pterocarpus marsupium Roxb. - A comprehensive review. Pharmacognosy Rev. 3: 359363.

Estrela J.M., Ortega A., Mena S., Rodriguez M.L., Asensi M. 2013. Pterostilbene: biomedical applications. Crit. Rev. Clin. Lab. Sci. 50: 65-78.

Gamborg O.L., Miller R.A., Ojima K. 1968. Nutrient requirements of suspension cultures of soybean root cells. Exp. Cell Res. 50: 
151-158.

Grover J.K., Vats V., Yadav, S.S. 2005. Pterocarpus marsupium extract (Vijayasar) prevented the alteration in metabolic patterns induced in the normal rat by feeding an adequate diet containing fructose as sole carbohydrate. Diabetes Obes. Metab. 7: 414-420.

Grover R.K., Maurya R., Roy R. 2004. Dynamic NMR investigation of two new interconvertible diasteriomeric epimers of natural 2-benzyl-2-hydroxybenzofuranone derivative from Pterocarpus marsupium. Tetrahedron 60: 2005-2010.

Gupta R., Gupta R.S. 2011. Effect of Pterocapus marsupium on streptozotocin-induced oxidative stress in kidney of diabetic wistar rats. J. Herbs. Spices Med. Plants 17: 169-182.

Gupta R., Gupta R.S. 2010. Hepatoprotective action of Pterocarpus marsupium against streptozotocin-induced oxidative stress. Egypt. J. Biol. 12: 44-51.

Gupta R., Gupta R.S. 2009. Effect of Pterocarpus marsupium in streptozotocin-induced hyperglycemic state in rats: comparison with glibenclamide. Diabetol. Croat. 38: 39-45.

Handa S.S., Singh R., Maurya R., Satti N.K., Suri K. A., Suri O.P. 2000. Pterocarposide, an isoaurone C-glucoside from Pterocarpus marsupium. Tetrahedron Lett. 41: 1579-1581.

Hari P.U., Gaikwad D.K. 2011. Pterocarpus marsupium: a valuable medicinal plant in diabetes management. Int. J. Appl. Biol. Pharm. Technol. 2: 6-13.

Hayward A.C., Tollenaere R., Dalton-Morgan J., Batley J. 2015. Molecular marker applications in plants. In: Batley J. (ed), Plant Genotyping Methods. Molecular Biology Volume 1245. Springer, New York, pp. 13-27.

Huang T., McDonald K.A. 2012. Bioreactor systems for in vitro production of foreign proteins using plant cell cultures. Biotechnol. Adv. 30: 398-409.

Husain M.K., Anis M., Shahzad A. 2010. Somatic embryogenesis and plant regeneration in Pterocarpus marsupium Roxb. Trees 24: 781-787.

Husain M.K., Anis M., Shahzad A. 2008. In vitro propagation of a multipurpose leguminous tree (Pterocarpus marsupium Roxb.) using nodal explants. Acta Physiol. Plant. 30: 353-359.

Husain M.K., Anis M., Shahzad A. 2007. In vitro propagation of Indian Kino (Pterocarpus marsupium Roxb.) using thidiazuron. In Vitro Cell. Dev. Biol. Plant 43: 59-64.

IUCN 2017. World Conservation Monitoring Centre 1998. Pterocarpus marsupium. IUCN Red List Threat. Species. Version 2017.2.

Jahromi M.A., Ray A.B. 1993. Antihyperlipidemic effect of flavonoids from Pterocarpus marsupium. J. Nat. Prod. 56: 989-994.

Jaiswal S., Arya S., Kant T. 2013. Role of various additives in controlling shoot tip necrosis of Pterocarpus marsupium Roxb. - a multipurpse leguminous tree. J. Phytol. Res. 26: 43-46.

Jaiswal S., Choudhary M., Arya S., Kant T. 2015. Micropropagation of adult tree of Pterocarpus marsupium Roxb. using nodal explants. J. Plant Dev. 22: 21-30.

Joshi K.R., Devkota H.P., Yahara S. 2012. Chemical analysis of heartwood of bijayasal (Pterocarpus marsupium Roxb.). Nepal J. Sci. Technol. 13: 219-224.

Kalia R.K., Rai M.K., Kalia S., Singh R., Dhawan A.K. 2011. Microsatellite markers: an overview of the recent progress in plants. Euphytica 177: 309-334.

Kher M.M., Nataraj M., Teixeira da Silva J.A. 2016. Micropropagation of Crataeva L. species. Rend. Lincei 27: 157-167.
Kulus D., Zalewska M. 2014. Cryopreservation as a tool used in long-term storage of ornamental species - a review. Sci. Hortic. 168: 88-107.

Leifert C., Morris C.E., Waites W.M. 1994. Ecology of microbial saprophytes and pathogens in tissue culture and field-grown plants: Reasons for contamination problems in vitro. Crit. Rev. Plant Sci. 13: 139-183.

Lloyd G., McCown B. 1980. Commercially-feasible micropropagation of mountain laurel, Kalmia latifolia, by use of shoottip culture. Int. Plant Propag. Soc. Proc. 30: 421-427.

Manickam M., Ramanathan M., Farboodniay Jahromi M.A., Chansouria J.P.N., Ray A.B. 1997. Antihyperglycemic activity of phenolics from Pterocarpus marsupium. J. Nat. Prod. 60: 609-610.

Mathew J., Rao A.V.S. 1984. Propterol: B, A further 1,3-diarylpropan-2-ol from Pterocarpus marsupium. Phytochemistry 23: 1814-1815.

Mathew J., Rao A.V.S., Rambhav S. 1984. Propterol - an antibacterial agent from Pterocarpus marsupium. Curr. Sci. 53: 576-577.

Mathew J., Rao A.V.S., Rao N.V.S. 1977. Photooxidation of pterostilbene from Pterocarpus marsupium Roxb. Curr. Sci. 46: 337-338.

Maurya R., Ray A.B., Chattopadhyay S.K., Duah F.K., Lin M.C., Slatkin D.J., Schiff P.L. 1985. The synthesis of propterol, a novel 1,3-diarylpropan-2-ol from Pterocarpus marsupium. J. Nat. Prod. 48: 313-315.

Maurya R., Singh R., Deepak M., Handa S.S., Yadav P.P., Mishra P.K. 2004. Constituents of Pterocarpus marsupium: An ayurvedic crude drug. Phytochemistry 65: 915-920.

McCormack D., McFadden D. 2013. A review of pterostilbene antioxidant activity and disease modification. Oxid. Med. Cell. Longev. 2013: 1-5.

Mishra A., Srivastava R., Srivastava S.P., Gautam S., Tamrakar A.K., Maurya R., Srivastava A.K. 2013. Antidiabetic activity of heart wood of Pterocarpus marsupium Roxb. and analysis of phytoconstituents. Indian J. Exp. Biol. 51: 363-374.

Mishra Y., Rawat R., Rana P.K., Sonkar M.K., Mohammad N. 2014. Effect of seed mass on emergence and seedling development in Pterocarpus marsupium Roxb. J. For. Res. 25: 415-418.

Mohankumar S.K., O'Shea T., McFarlane J.R. 2012. Insulinotrophic and insulin-like effects of a high molecular weight aqueous extract of Pterocarpus marsupium Roxb. hardwood. J. Ethnopharmacol. 141: 72-79.

Muller F., Vaillant A., Bâ A., Bouvet J.M. 2006. Isolation and characterization of microsatellite markers in Pterocarpus officinalis Jacq. Mol. Ecol. Notes 6: 462-464.

Murashige T., Skoog F. 1962. A revised medium for rapid growth and bioassays with tobacco tissue cultures. Physiol. Plant. 15: 473-497.

Nataraj M., Kher M.M., Teixeira da Silva J.A. 2016. Micropropagation of Clerodendrum L. species: a review. Rend. Lincei 27: 169-179.

Nhut D.T., Teixeira da Silva J.A., Aswath C.R. 2003. The importance of the explant on regeneration in thin cell layer technology. In Vitro Cell. Dev. Biol. Plant 39: 266-276.

Patel A., Patel I., 2016. Effect of seed direction and growth media on in vitro seeds germination and seedling establishment of Pterocarpus marsupium Roxb. Int. J. Plant, Anim. Environ. Sci. 6: 139-145.

Porika M., Tippani R., Mamidala P., Peddaboina V., Thamidala C., Abbagani S., Nanna R.S. 2009. Micropropagation of red kino tree (Pterocarpus marsupium Roxb.): a medicinally important 
plant. Int. J. Plant Dev. Biol. 3: 52-55.

Rai M.K., Asthana P., Singh S.K., Jaiswal V.S., Jaiswal U., Kant S. 2009. The encapsulation technology in fruit plants - a review. Biotechnol. Adv. 27: 671-679.

Rai M.K., Shekhawat N.S. 2014. Recent advances in genetic engineering for improvement of fruit crops. Plant Cell Tissue Organ Cult. 116: 1-15.

Rao A.V.S., Mathew J., Sankaram A.V.B. 1984. Propterol: a 1,3-diarylpropan-2-ol from Pterocarpus marsupium. Phytochemistry 23: 897-898.

Rivera-Ocasio E., Aide T.M., McMillan W.O. 2006. The influence of spatial scale on the genetic structure of a widespread tropical wetland tree, Pterocarpus officinalis (Fabaceae). Conserv. Genet. 7: 251-266.

Rivera-Ocasio E., Aide T.M., McMillan W.O. 2002. Patterns of genetic diversity and biogeographical history of the tropical wetland tree, Pterocarpus officinalis (Jacq.), in the Caribbean basin. Mol. Ecol. 11: 675-683.

Ruffoni B., Savona M. 2013. Physiological and biochemical analysis of growth abnormalities associated with plant tissue culture. Hortic. Environ. Biotechnol. 54: 191-205.

Sanatombi R., Sanatombi K. 2017. Biotechnology of Zingiber montanum (Koenig) Link ex A. Dietr.: A review. J. Appl. Res. Med. Aromat. Plants 4: 1-4.

Saslis-Lagoudakis C.H., Klitgaard B.B., Forest F., Francis L., Savolainen V., Williamson E.M., Hawkins J.A. 2011. The use of phylogeny to interpret cross-cultural patterns in plant use and guide medicinal plant discovery: An example from Pterocarpus (leguminosae). PLoS One 6: e22275.

Schlötterer C. 2004. The evolution of molecular markers - just a matter of fashion? Nat. Rev. Genet. 5: 63-69.

Sharma A.K., Sharma M.K. 2009. Plants as bioreactors: Recent developments and emerging opportunities. Biotechnol. Adv. 27: 811-32.

Sharma S., Shahzad A., Teixeira da Silva J.A. 2013. Synseed technology - a complete synthesis. Biotechnol. Adv. 31: 186207.

Sheehan E.W., Zemaitis M.A., Slatkin D.J., Schiff P.L. 1983. A constituent of Pterocarpus marsupium, (-)-epicatechin, as a potential antidiabetic agent. J. Nat. Prod. 46: 232-234.

Teixeira da Silva J.A. 2012a. The role of thin cell layers in regeneration and transformation in orchids. Plant Cell. Tissue Organ Cult. 113: 149-161.

Teixeira da Silva J.A. 2012b. Is BA (6-benzyladenine) bap (6-benzylaminopurine)? Asian Australasian J. Plant Sci. Biotechnol. 6: 121-124.

Teixeira da Silva J.A. 2012c. Callus, calluses or calli: multiple plurals? Asian Australasian J. Plant Sci. Biotechnol. 6: 125-126.

Teixeira da Silva J.A. 2003. Thin cell layer technology in ornamental plant micropropagation and biotechnology.
African J. Biotechnol. 2: 683-691.

Teixeira da Silva J.A., Bolibok H., Rakoczy-Trojanowska M. 2007a. Molecular markers in micropropagation, tissue culture and in vitro plant research. Genes Genomes Genom. 1: 66-72.

Teixeira da Silva J.A., Dobránszki J.2014. Dissecting the concept of the thin cell layer: theoretical basis and practical application of the plant growth correction factor to apple, Cymbidium and chrysanthemum. J. Plant Growth Regul. 33: 881-895.

Teixeira da Silva J.A., Dobránszki J. 2013. Plant thin cell layers: a 40-year celebration. J. Plant Growth Regul. 32: 922-943.

Teixeira da Silva J.A., Dobránszki J., Ross S. 2013. Phloroglucinol in plant tissue culture. In Vitro Cell. Dev. Biol. Plant 49: 1-16.

Teixeira da Silva J.A., Kim H., Engelmann F. 2015. Chrysanthemum low-temperature storage and cryopreservation: a review. Plant Cell. Tissue Organ Cult. 120: 423-440.

Teixeira da Silva J.A., Malabadi R.B. 2012. Factors affecting somatic embryogenesis in conifers. J. For. Res. 23: 503-515.

Teixeira da Silva J.A., Tran Thanh Van K., Stefania B., Nhut D.T., Altamura M.M. 2007b. Thin cell layers: developmental building blocks in ornamental biotechnology. Floric. Ornam. Biotechnol. 1: 1-13.

Teixeira da Silva J.A., Zeng S., Wicaksono A., Kher M.M., Kim H., Hosokawa M., Dewir Y.H., 2017. In vitro propagation of African violet: a review. South African J. Bot. 112: 501-507.

The Plant List 2018. Pterocarpus. http://www.theplantlist.org/1.1/ browse/A/Leguminosae/Pterocarpus/ (last accessed 6.2.2018).

Thimijan R.W., Heins R.D. 1983. Photometric, radiometric, and quantum light units of measure: a review of procedures for interconversion. HortScience 18: 818-822.

Tippani R., Vemunoori A.K., Yarra R., Nanna R.S., Abbagani S., Thammidala C. 2013a. Adventitious shoot regeneration from immature zygotic embryos of Indian Kino tree (Pterocarpus marsupium Roxb.) and genetic integrity analysis of in vitro derived plants using ISSR markers. Hortic. Environ. Biotechnol. 54: 531-537.

Tippani R., Yarra R., Bulle M., Porika M., Abbagani S., Thammidala C. 2013b. In vitro plantlet regeneration and Agrobacterium tumefaciens-mediated genetic transformation of Indian Kino tree (Pterocarpus marsupium Roxb.). Acta Physiol. Plant. 35 : 3437-3446.

Tiwari S., Shah P., Singh K. 2004. In vitro propagation of Pterocarpus marsupium Roxb.: an endangered medicinal tree. Indian J. Biotechnol. 3: 422-425.

White P.R. 1963. The Cultivation of Animal and Plant Cells. $2^{\text {nd }}$ Ed. Ronald Press, NY, pp. 239-252.

Xiao Y., Niu G., Kozai T. 2010. Development and application of photoautotrophic micropropagation plant system. Plant Cell Tissue Organ Cult. 105: 149-158.

Ziv M. 2005. Simple bioreactors for mass propagation of plants. Plant Cell Tissue Organ Cult. 81: 277-285. 